PAPER

Highly efficient one-step synthesis of carbon encapsulated nanocrystals by the oxidation of metal $\pi$-complexes

To cite this article: Boyang Liu et al 2017 Nanotechnology 28325603

View the article online for updates and enhancements.
Related content

- Low temperature in situ synthesis and formation mechanism of carbon encapsulated various nanocrystals by electrophilic oxidation of metallocene complexes

Boyang Liu, Chunhua Fan, Jianwei Chen et al.

Synthesis of binary iron-carbon nanoparticles by UV laser photolysis of $\mathrm{Fe}(\mathrm{CO}) 5$ with various hydrocarbons A V Eremin, E V Gurentsov and S A Musikhin

Biomolecule-assisted synthesis of $\operatorname{In}(\mathrm{OH}) 3$ nanocubes and In2O3 nanoparticles: photocatalytic degradation of organic contaminants and $\mathrm{CO}$ oxidation Arpan Kumar Nayak, Seungwon Lee, Youngku Sohn et al. 


\title{
Highly efficient one-step synthesis of carbon encapsulated nanocrystals by the oxidation of metal $\pi$-complexes
}

\author{
Boyang Liu ${ }^{1,4}$, Yingfeng Shao ${ }^{2,4}$, Xin Xiang ${ }^{1}$, Fuhua Zhang ${ }^{1}$, \\ Shengchang Yan $^{1}$ and Wenge $\mathrm{Li}^{3}$ \\ ${ }^{1}$ College of Ocean Science and Engineering, Shanghai Maritime University, Shanghai 201306, People's \\ Republic of China \\ ${ }^{2}$ State Key Laboratory of Nonlinear Mechanics, Institute of Mechanics, Beijing 100190, People's Republic \\ of China \\ ${ }^{3}$ Merchant Marine College, Shanghai Maritime University, Shanghai 201306, People's Republic of China \\ E-mail: byliu@shmtu.edu.cn, shaoyf@lnm.imech.ac.cn, 2243366720@qq.com, fhzhang@ shmtu.edu.cn, \\ yanshengchang123@qq.com andwgli@shmtu.edu.cn
}

Received 11 April 2017, revised 8 June 2017

Accepted for publication 14 June 2017

Published 19 July 2017

\begin{abstract}
Various carbon encapsulated nanocrystals, including $\mathrm{MnS}$ and $\mathrm{MnO}, \mathrm{Cr}_{2} \mathrm{O}_{3}, \mathrm{MoO}_{2}, \mathrm{Fe}_{7} \mathrm{~S}_{8}$ and $\mathrm{Fe}_{3} \mathrm{O}_{4}$, and $\mathrm{ZrO}_{2}$, are prepared in one step and in situ by a simple and highly efficient synthesis approach. The nanocrystals have an equiaxed morphology and a median size smaller than $30 \mathrm{~nm}$. Tens and hundreds of these nanocrystals are entirely encapsulated by a wormlike amorphous carbon shell. The formation of a core-shell structure depends on the strongly exothermic reaction of metal $\pi$-complexes with ammonium persulfate in an autoclave at below $200{ }^{\circ} \mathrm{C}$. During the oxidation process, the generated significant amounts of heat will destroy the molecular structure of the metal $\pi$-complex and cleave the ligands into small carbon fragments, which further transform into an amorphous carbon shell. The central metal atoms are oxidized to metal oxide/ sulfide nanocrystals. The formation of a core-shell structure is independent of the numbers of ligands and carbon atoms as well as the metal types, implying that any metal $\pi$-complex can serve as a precursor and that various carbon encapsulated nanocrystals can be synthesized by this method.
\end{abstract}

Supplementary material for this article is available online

Keywords: carbon encapsulated nanocrystals, core-shell, low temperature synthesis, metal $\pi$ complex

(Some figures may appear in colour only in the online journal)

\section{Introduction}

The core-shell structure can generally be defined as comprising an inner core and an external shell with different chemical compositions. The unique structural feature allows the possibility of combining desirable properties of diverse materials and thereby improving physical and chemical properties compared to their single-component counterparts.

\footnotetext{
${ }^{4}$ Author to whom any correspondence should be addressed.
}

Hence, various types of core-shell structures have attracted increasing research interest because of their potentially broad range of applications for drug delivery, bioimaging, sensors and catalysis [1]. Among them, carbon encapsulated nanocrystals are one of the most common classes. This is largely ascribed to the high chemical inertness, ease of surface modification, good conductivity and tensile strength of the carbon shell [2-4].

On the basis of the conventional preparation techniques for carbon nanomaterials, a number of synthetic routes to 
Table 1. Synthesis conditions of carbon encapsulated nanocrystals.

\begin{tabular}{|c|c|c|c|c|c|c|c|c|}
\hline \multirow[b]{2}{*}{ No. } & \multicolumn{4}{|c|}{ Reactants } & \multirow[b]{2}{*}{$\begin{array}{l}\text { Vol. of auto- } \\
\text { clave (ml) }\end{array}$} & \multirow[b]{2}{*}{$\begin{array}{l}\text { Temp. } \\
\left({ }^{\circ} \mathrm{C}\right)\end{array}$} & \multirow[b]{2}{*}{$\begin{array}{l}\text { Time } \\
(\mathrm{min})\end{array}$} & \multirow[b]{2}{*}{ Major products } \\
\hline & Metal $\pi$-complex & $\mathrm{mmol}$ & $\begin{array}{l}\text { Ammonium } \\
\text { persulphate }\end{array}$ & mmol & & & & \\
\hline$\# 1$ & $\left(\mathrm{C}_{5} \mathrm{H}_{5}\right) \mathrm{Mn}(\mathrm{CO})_{3}$ & 5 & $\left(\mathrm{NH}_{4}\right)_{2} \mathrm{~S}_{2} \mathrm{O}_{8}$ & 10 & & & & $\mathrm{MnS}$ and $\mathrm{MnO} @ \mathrm{C}$ \\
\hline$\# 2$ & $\left(\mathrm{C}_{6} \mathrm{H}_{6}\right) \mathrm{Cr}(\mathrm{CO})_{3}$ & 5 & & 10 & & & & $\mathrm{Cr}_{2} \mathrm{O}_{3} @ \mathrm{C}$ \\
\hline \#3 & $\left(\mathrm{C}_{7} \mathrm{H}_{8}\right) \mathrm{Mo}(\mathrm{CO})_{3}$ & 5 & & 10 & 50 & 200 & 30 & $\mathrm{MoO}_{2} @ \mathrm{C}$ \\
\hline$\# 4$ & $\left(\mathrm{C}_{8} \mathrm{H}_{8}\right) \mathrm{Fe}(\mathrm{CO})_{3}$ & 2 & & 8 & & & & $\begin{array}{l}\mathrm{Fe}_{7} \mathrm{~S}_{8} \text { and } \\
\mathrm{Fe}_{3} \mathrm{O}_{4} @ \mathrm{C}\end{array}$ \\
\hline$\# 5$ & $\left(\mathrm{C}_{9} \mathrm{H}_{7}\right)_{2} \mathrm{ZrCl}_{2}$ & 2 & & 4 & & & & $\mathrm{ZrO}_{2} @ \mathrm{C}$ \\
\hline
\end{tabular}

these kinds of nanocomposites have been developed over decades. The arc discharge and laser ablation techniques can produce very high temperatures of about $3000 \mathrm{~K}$ to evaporate the metal/carbon composite and then effectively form carbonencapsulated metal or metal carbide nanocrystals [5, 6]. Thermal decomposition of carbon-containing precursors through chemical vapor deposition, pyrolysis/carbonization process and flame spray synthesis is commonly employed to achieve a carbon layer on a variety of preformed cores and cores formed in situ [7-9]. These processes all involve hightemperature treatment, a vacuum/inert atmosphere, and specific facilities. A hydrothermal carbonization process for a core-shell structure can be carried out in an autoclave at a low temperature of $140-200{ }^{\circ} \mathrm{C}$, using different types of saccharides as carbon precursor [10]. For example, Au-cored carbon spheres could be hydrothermally prepared at $180{ }^{\circ} \mathrm{C}$ for $4-20 \mathrm{~h}$ from an aqueous glucose solution containing $\mathrm{HAuCl}_{4}$ [11]. Additionally, elliptical $\mathrm{Fe}_{3} \mathrm{O}_{4} / \mathrm{C}$ core-shell nanorings were prepared by a multi-step hydrothermal route at $180{ }^{\circ} \mathrm{C}$ for $9-18 \mathrm{~h}$ using iron glycolate nanosheets as templates [12]. $\mathrm{LiFePO}_{4} / \mathrm{C}$ submicron rods were also synthesized via a hydrothermal process at $200{ }^{\circ} \mathrm{C}$ for $10.5 \mathrm{~h}$ following heat treatment in an $\mathrm{N}_{2}$ atmosphere at $500{ }^{\circ} \mathrm{C}$ for $2 \mathrm{~h}$ [13]. Although the synthesis temperature is low, a hydrothermal treatment time as long as tens of hours and multiple steps are usually required to form the carbon shell, which is time-consuming and inefficient. It is therefore worth developing an alternative method featuring simple experimental procedures and providing a fast and reliable protocol for the synthesis of carbon encapsulated nanocrystals.

Our previous study proposed a general in situ synthesis approach for different carbon encapsulated oxide/sulfide nanocrystals with a core-shell structure by a solid-state explosive reaction between metallocene complexes and highoxidative ammonium salts in an autoclave at $200{ }^{\circ} \mathrm{C}[14,15]$. Compared to the conventional preparation techniques, the oxidizing agent is indispensable for the formation of carbon [16], implying that the effect of the presence of oxygen in the reaction apparatus on the product can be negligible, and that only a sealed reactor is sufficient for the synthesis. As no other specific apparatus or operating steps are needed, this method shows distinct advantages such as its simple procedure, low temperature, and high efficiency and yield, which is attractive for lithium-ion batteries [17-19]. Until now, most metallocene complexes, typically consisting of cyclopentadienyl ligand with the formula $\mathrm{C}_{5} \mathrm{H}_{5}$ bound to central metal atoms, have been proved to be promising precursors for the preparation of carbon encapsulated nanocrystals by this method. Although the cyclopentadienyl ligand is among the most frequently utilized $\pi$ ligand in organometallic chemistry, other cyclic ligands such as benzene $\left(\mathrm{C}_{6} \mathrm{H}_{6}\right)$, cycloheptatriene $\left(\mathrm{C}_{7} \mathrm{H}_{8}\right)$, cyclooctatetraene $\left(\mathrm{C}_{8} \mathrm{H}_{8}\right)$ and indenyl $\left(\mathrm{C}_{9} \mathrm{H}_{7}\right)$ can serve as metal-binding platforms as well, to form metal $\pi$ complexes. These complexes have similar electronic structures and properties to metallocenes, which are conceived to undergo the same oxidation process to produce carbon encapsulated nanocrystals. Consequently, in this work, several metal $\pi$-complexes including cyclopentadienyl manganese tricarbonyl $\left(\left(\mathrm{C}_{5} \mathrm{H}_{5}\right) \mathrm{Mn}(\mathrm{CO})_{3}\right)$, benzene chromium tricarbonyl $\left(\left(\mathrm{C}_{6} \mathrm{H}_{6}\right) \mathrm{Cr}(\mathrm{CO})_{3}\right)$, cycloheptatriene molybdenum tricarbonyl $\left(\left(\mathrm{C}_{7} \mathrm{H}_{8}\right) \mathrm{Mo}(\mathrm{CO})_{3}\right)$, cyclooctatetraene iron tricarbonyl $\left(\left(\mathrm{C}_{8} \mathrm{H}_{8}\right) \mathrm{Fe}(\mathrm{CO})_{3}\right)$ and bis(indenyl)zirconium dichloride $\left(\left(\mathrm{C}_{9} \mathrm{H}_{7}\right)_{2} \mathrm{ZrCl}_{2}\right)$ are used to react with ammonium persulfate $\left(\left(\mathrm{NH}_{4}\right)_{2} \mathrm{~S}_{2} \mathrm{O}_{8}\right)$, and various carbon encapsulated nanocrystals with core-shell structures are successfully fabricated (denoted as 'formula of inner core'@C, such as MnS and $\mathrm{MnO} @ \mathrm{C}, \mathrm{Cr}_{2} \mathrm{O}_{3} @ \mathrm{C}$, etc).

\section{Experimental details}

\subsection{Materials and preparation}

All the chemicals, of analytically pure grade, were received from Amethyst Chemicals (J\&K Scientific Ltd, China). Several carbon encapsulated nanocrystals were prepared by the oxidation of metal $\pi$-complexes in an autoclave, and the synthesis conditions are presented in table 1 . Typically, the metal $\pi$-complex and $\left(\mathrm{NH}_{4}\right)_{2} \mathrm{~S}_{2} \mathrm{O}_{8}$ were roughly milled in a mortar and subsequently heated at $200{ }^{\circ} \mathrm{C}$ for $30 \mathrm{~min}$. After cooling to room temperature, the as-prepared black powder was washed with deionized water and carbon encapsulated nanocrystals were eventually obtained after drying overnight at $120^{\circ} \mathrm{C}$. It is worth noting that the simple and highly efficient synthesis process performed in a sealed reactor is based on the rapid reaction of metal $\pi$-complexes with $\left(\mathrm{NH}_{4}\right)_{2} \mathrm{~S}_{2} \mathrm{O}_{8}$ without any solvent, which is completely different than the hydrothermal method depending on high solvent vapour pressure and long reaction times. The purpose of the use of 

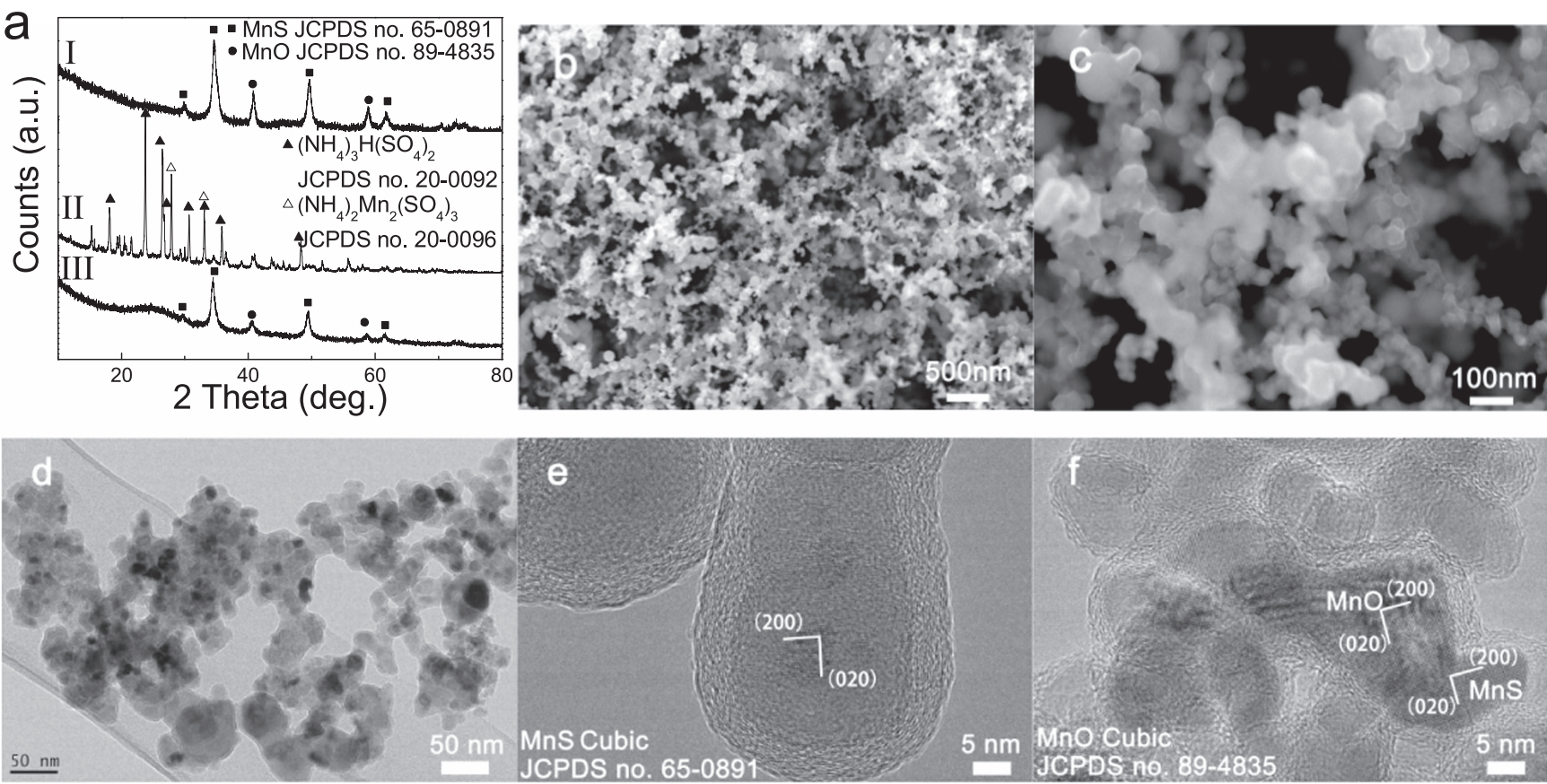

Figure 1. (a) XRD patterns of the powder in sample \#1 in different positions in the autoclave: (I) top; (II) bottom; (III) washed bottom. (b), (c) SEM images of the top powder at different magnifications. (d)-(f) TEM and HRTEM images of the top powder.

the autoclave was to prevent the escape of vaporized metal $\pi$ complexes before the reaction temperature and the gaseous intermediate products.

\subsection{Characterization}

X-ray diffraction (XRD) was performed on a PANalytical X'Pert PRO diffractometer with $\mathrm{Cu} \mathrm{K}_{\alpha}$ radiation for phase identification. A Raman spectrum was recorded by a Bruker Senterra micro Raman spectrometer with an excitation wavelength of $633 \mathrm{~nm}$ at $2 \mathrm{~mW}$. The morphology and microstructure of the samples were analyzed by scanning electron microscopy (SEM, JEOL JSM 7500F) and transmission electron microscopy (TEM, JEOL JEM 2010) equipped with an X-ray energy-dispersive spectroscopy system (EDS, EDAX) for composition analysis. Differential scanning calorimetry (DSC) was carried out on a Netzsch DSC $204 \mathrm{~F} 1$ at a heating rate of $10{ }^{\circ} \mathrm{C} \mathrm{min}-1$ using a sealed aluminum pan.

\section{Results and discussion}

The macroscopic morphology of as-prepared sample \#1 shows an obvious stratification phenomenon. Fluffy powder and soft powder agglomerates are yielded in the top and bottom of the autoclave, respectively. The XRD patterns in figure 1(a) show that the $\mathrm{MnS}$ and $\mathrm{MnO}$ are the major phases in the top powder, while the $\left(\mathrm{NH}_{4}\right)_{3} \mathrm{H}\left(\mathrm{SO}_{4}\right)_{2}$ and $\left(\mathrm{NH}_{4}\right)_{2} \mathrm{Mn}_{2}\left(\mathrm{SO}_{4}\right)_{3}$ reside in the bottom powder. When these soluble ammonium salts are eliminated by the washing process, the $\mathrm{MnS}$ and $\mathrm{MnO}$ diffraction peaks become visible, which is identical with the top powder. The carbon diffraction peaks are not evident because of its poor crystallinity at low synthesis temperature. The core-shell structure can be directly observed by the SEM images of the top powder, consisting of interconnected nanoparticles with obvious white cores inside (figures 1(b) and (c)). The TEM and high-resolution TEM (HRTEM) images in figures 1(d)-(f) indicate that the turbostratic carbon shell encapsulated tens of $\mathrm{MnS}$ and $\mathrm{MnO}$ nanocrystals as a whole. The enlarged HRTEM images with clear lattice fringes are shown in the supplementary data (figure S1). These small nanocrystals have a diameter range of 2-32 $\mathrm{nm}$ according to the TEM measurement and their median size is $13.34 \mathrm{~nm}$ (figure S2). The bottom powder mainly contains core-shell structure despite the emergence of some irregularly-shaped carbon particles (figure S3). In addition, some $\left(\mathrm{NH}_{4}\right)_{2} \mathrm{Mn}_{2}\left(\mathrm{SO}_{4}\right)_{3}$ nanocrystals can also be encapsulated by the carbon shell (figure S4).

In sample \#2, only the fluffy top powder is obtained and its XRD pattern reveals that $\mathrm{Cr}_{2} \mathrm{O}_{3}$ is formed during the oxidation synthesis (figure 2(a)). Under SEM examination, the homogeneous nanoparticles exhibit an equiaxed morphology and aggregate together. However, the semitransparent carbon layer cannot be seen on the surface of these nanoparticles (figures 2(b) and (c)). Thus, Raman spectroscopy is carried out and two typical D and G bands of amorphous carbon are represented, confirming the existence of carbon shell in the specimen (figure S5). The TEM image further displays that the $\mathrm{Cr}_{2} \mathrm{O}_{3}$ nanocrystals are encapsulated by a very thin carbon shell (figure 2(d)). Single-crystal lattices of one typical $\mathrm{Cr}_{2} \mathrm{O}_{3}$ nanocrystal can be identified in the enlarged HRTEM image (inset of figure 2(d); the enlarged image is shown in figure S6), and the thickness of the carbon shell is only about $0.5 \mathrm{~nm}$. The diameter of $\mathrm{Cr}_{2} \mathrm{O}_{3}$ nanocrystals is less than $45 \mathrm{~nm}$ and the median size is calculated to 

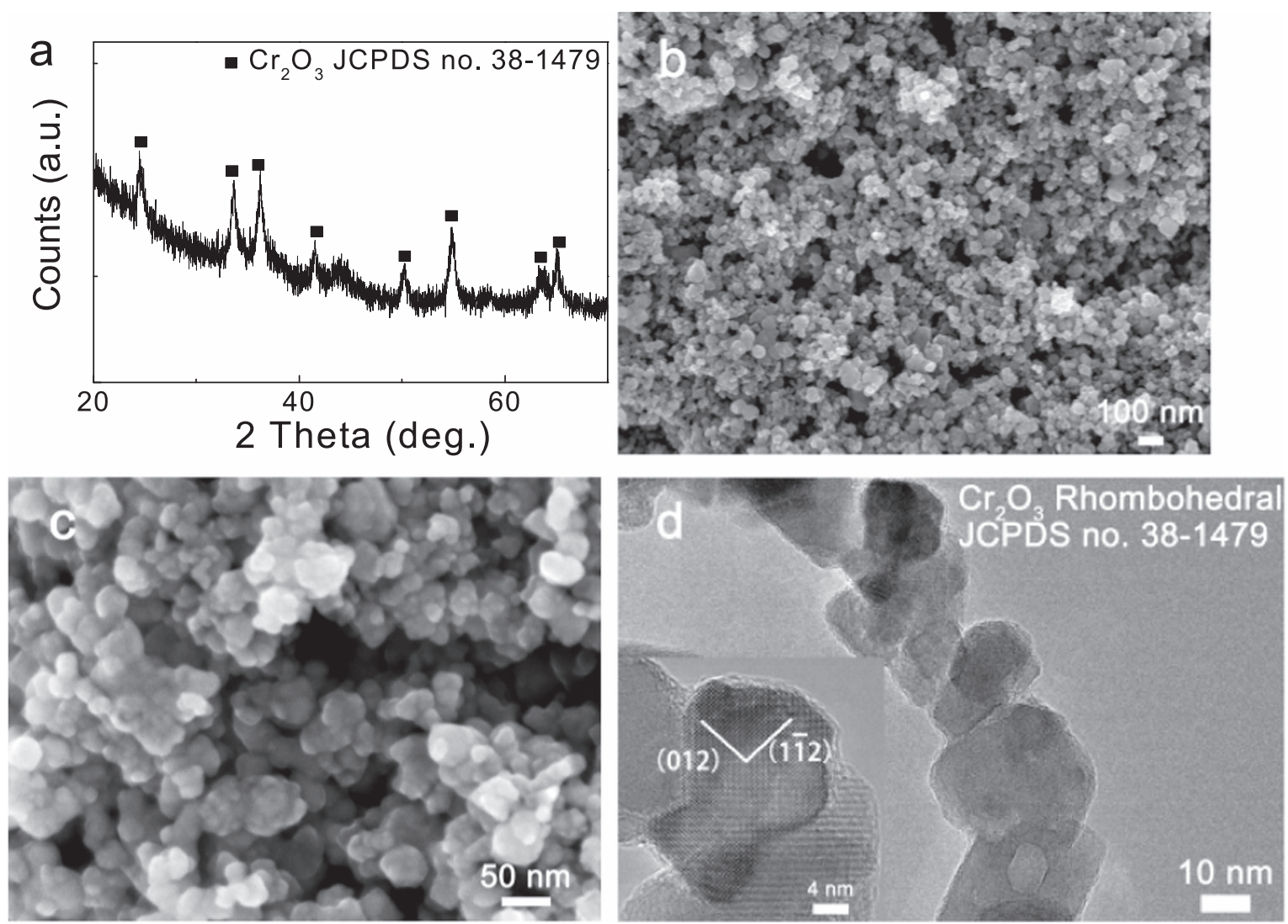

Figure 2. Characterization of $\mathrm{Cr}_{2} \mathrm{O}_{3} @ \mathrm{C}$ : (a) XRD pattern; (b), (c) SEM images; (d) TEM image. Inset is the HRTEM image of the lattice fringes of an individual $\mathrm{Cr}_{2} \mathrm{O}_{3}$ nanocrystal encapsulated by a thin carbon layer.
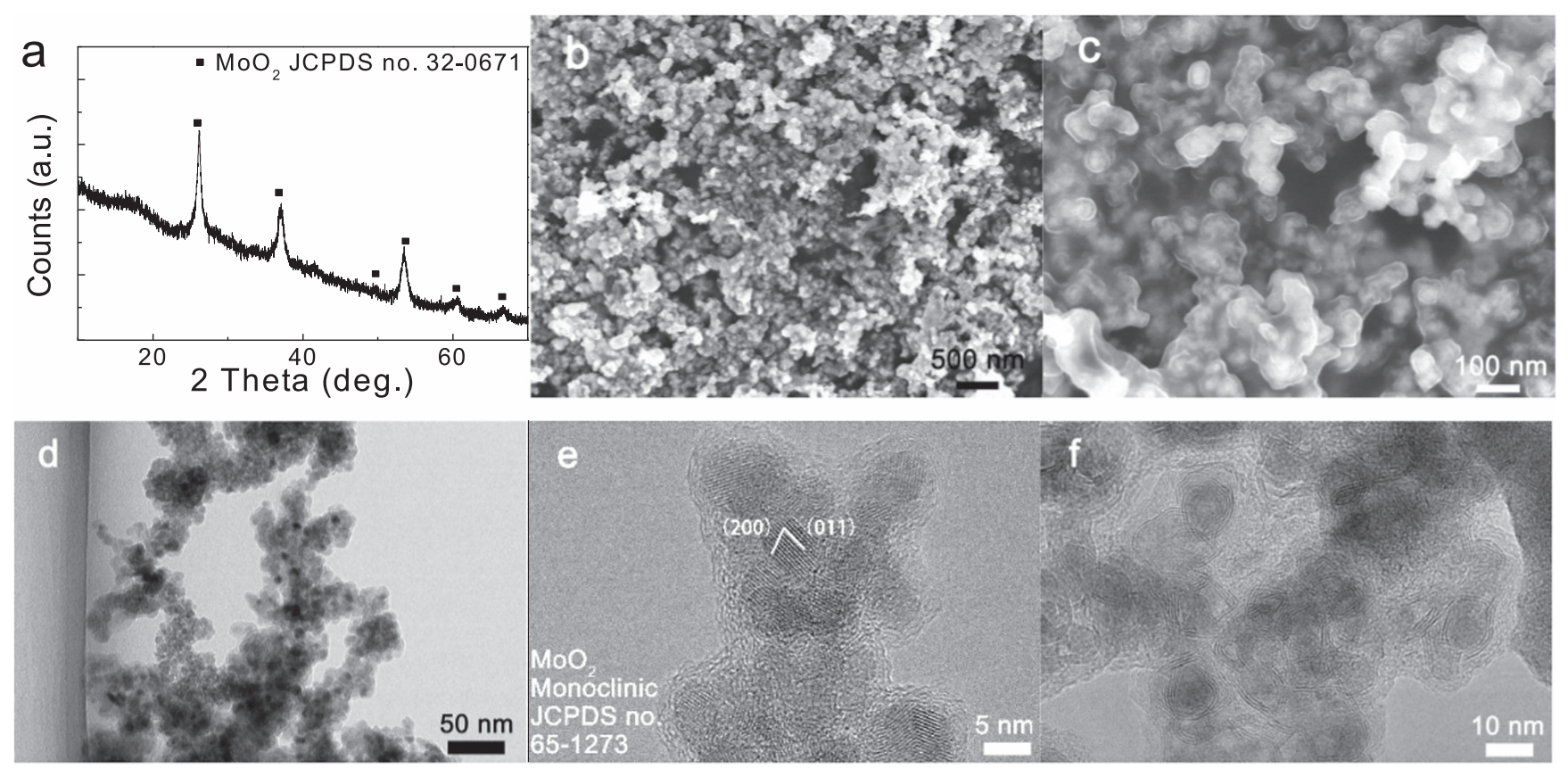

Figure 3. Characterization of $\mathrm{MoO}_{2} @ \mathrm{C}$ : (a) XRD pattern; (b), (c) SEM images; (d)-(f) TEM and HRTEM images.

be $15.51 \mathrm{~nm}$, according to the size distribution histogram (figure S7).

Similar to sample \#2, only the fluffy powder can be found in the top of the autoclave in sample \#3 and the XRD pattern shows that the as-prepared powder is predominantly composed of $\mathrm{MoO}_{2}$ (figure 3(a)). SEM images indicate that the powder has a homogeneous morphology of nanoparticles, consisting of white cores under the complete coverage of a carbon layer (figures 3(b) and (c)). In fact, it can be seen under TEM observation that these cores are the agglomerate 

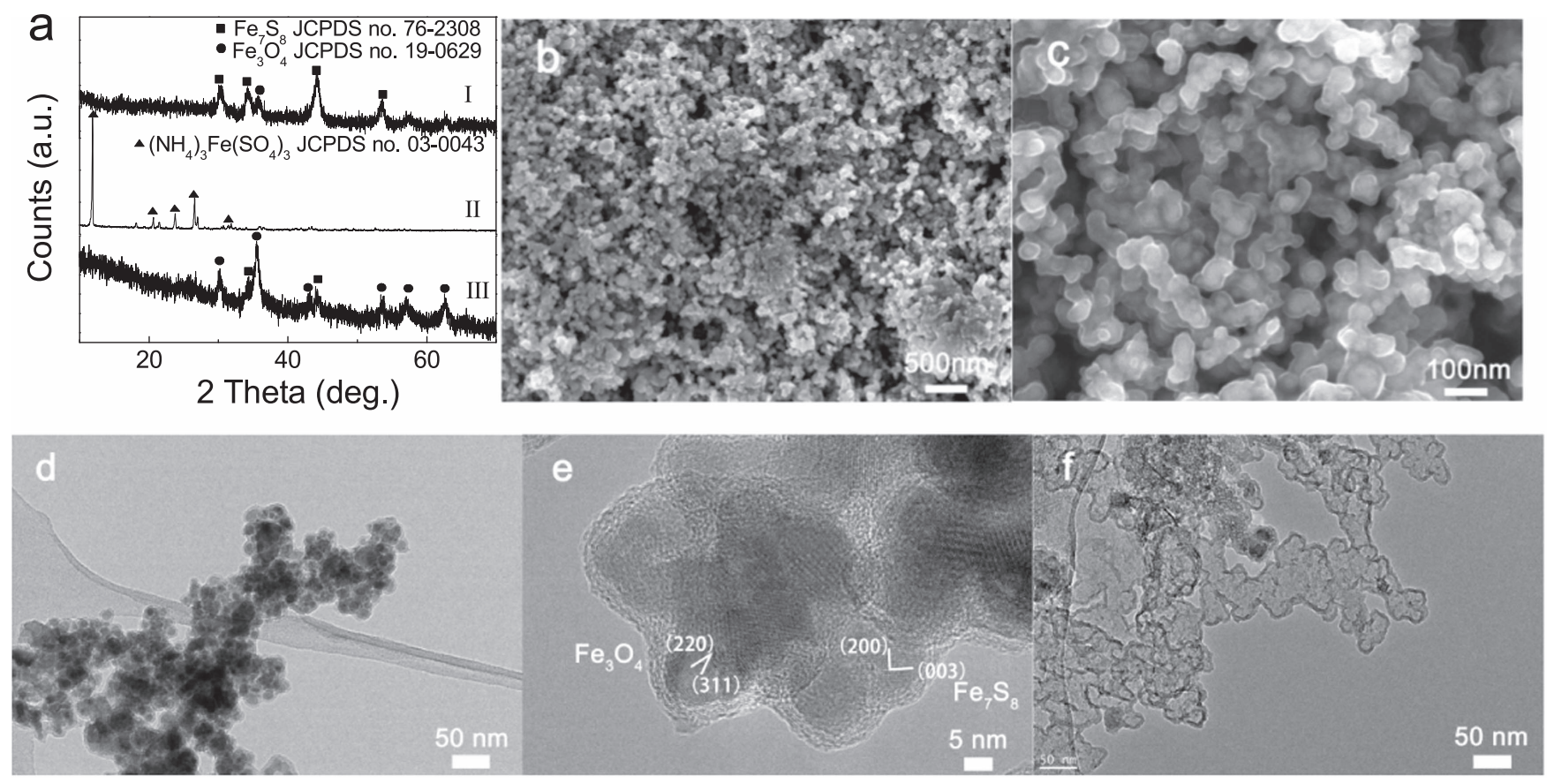

Figure 4. (a) XRD patterns of the powder in sample \#4 in different positions in the autoclave: (I) top; (II) bottom; (III) washed bottom. (b), (c) SEM images of the top powder at different magnifications. (d), (e) TEM and HRTEM images of the top powder. (f) TEM image of the washed bottom powder.

of quantities of ultrafine $\mathrm{MoO}_{2}$ nanocrystals and the thickness of the amorphous carbon shell is about $2 \sim 3 \mathrm{~nm}$ (figures 3(d) and (e); an enlarged figure $3(\mathrm{e})$ is shown in figure $\mathrm{S} 8$ ). These $\mathrm{MoO}_{2}$ nanocrystals have a typical diameter of less than $20 \mathrm{~nm}$ and a median size of $6.41 \mathrm{~nm}$ (figure S9). Moreover, it is worth mentioning that the $\mathrm{MoO}_{2}$ nanocrystals coexist with a small amount of $\mathrm{MoS}_{2}$ folded into a few layers (figure 3(f)). The formation mechanism has been discussed elsewhere [18].

For sample \#4, powders with different macroscopic morphologies are separately collected from the different positions of the autoclave. As shown in the XRD patterns in figure 4(a), the top fluffy powder contains a mixture of phases of $\mathrm{Fe}_{7} \mathrm{~S}_{8}$ and $\mathrm{Fe}_{3} \mathrm{O}_{4}$, whereas the bottom soft agglomerates are abundant in $\left(\mathrm{NH}_{4}\right)_{3} \mathrm{Fe}\left(\mathrm{SO}_{4}\right)_{3}$. This soluble ammonium salt will be removed during the washing process, leaving behind the phases of $\mathrm{Fe}_{7} \mathrm{~S}_{8}$ and $\mathrm{Fe}_{3} \mathrm{O}_{4}$, which is the same as the top powder. The top powder also shows a uniform core-shell structure and both $\mathrm{Fe}_{7} \mathrm{~S}_{8}$ and $\mathrm{Fe}_{3} \mathrm{O}_{4}$ equiaxed nanocrystals are encapsulated by a thin amorphous carbon shell of about 3-5 nm, demonstrated by the SEM and TEM images (figures 4(b)-(e); an enlarged figure 4(e) is shown in figure S10). Most of these nanocrystals have a narrow diameter distribution of 5-25 nm and a median size of $12.6 \mathrm{~nm}$ (figure S11). The SEM images of the washed bottom powder also show an evident core-shell structure. However, there is a large amount of hollows in the powder, which should be ascribed to the dissolution of interior $\left(\mathrm{NH}_{4}\right)_{3} \mathrm{Fe}\left(\mathrm{SO}_{4}\right)_{3}$ nanocrystals (figures S12 and 4(f)).

According to the XRD patterns of sample \#5 (figure 5(a)), besides the tetragonal phase of $\mathrm{ZrO}_{2}\left(\mathrm{t}-\mathrm{ZrO}_{2}\right)$, $\mathrm{NH}_{4} \mathrm{Cl}$ is formed in the top powder because of the combination of the chlorine in the precursor with ammonium in
$\left(\mathrm{NH}_{4}\right)_{2} \mathrm{~S}_{2} \mathrm{O}_{8}$. This byproduct can also be found in the bottom powder, together with some unknown product which may be supposed to be ammonium zirconium sulfate due to its good solubility in water, and the washed bottom powder is mainly composed of $\mathrm{t}-\mathrm{ZrO}_{2}$. SEM images of the top powder show that the nanoparticles are synthesized and that some of them have white cores, corresponding to the core-shell structure (figures 5(b) and (c)). TEM images clearly show the coreshell structure of carbon encapsulated equiaxed $\mathrm{ZrO}_{2}$ nanocrystals about $20-35 \mathrm{~nm}$ in diameter and an amorphous carbon shell about 20-30 nm in thickness (figures 5(d) and (e); an enlarged figure 5(e) is shown in figure S13). There are some smaller $\mathrm{t}-\mathrm{ZrO}_{2}$ nanocrystals ranging from 5 to $15 \mathrm{~nm}$ embedded inside the amorphous wormlike carbon (figure $\mathrm{S} 14)$. The size distribution histogram of the $\mathrm{ZrO}_{2}$ nanocrystals is shown in figure S15 and the median size is $22.57 \mathrm{~nm}$. It should be noted that a certain amount of necklace-like solid carbon is simultaneously formed during the synthesis and can be regarded as amorphous owing to the disordered lattice fringes revealed in the magnified image (figure 5(f)). The formation of a thick carbon shell and solid carbon should be attributed to the two $\mathrm{C}_{9} \mathrm{H}_{7}$ ligands in the precursor, in which the carbon content is relatively high with respect to $\mathrm{Zr}$. The morphology of the washed bottom powder in sample \#5 is not uniform, as irregularly-shaped carbonaceous materials can be found together with the core-shell structure (figure S16).

DSC curves of these samples using sealed pans were recorded in order to investigate the reaction behavior of the starting materials, as shown in figure 6 . The metal $\pi$-complexes can be very stable at low temperature, even with the presence of the strong oxidizing agent, and their following oxidation takes place at $100-200{ }^{\circ} \mathrm{C}$ on the basis of the very 

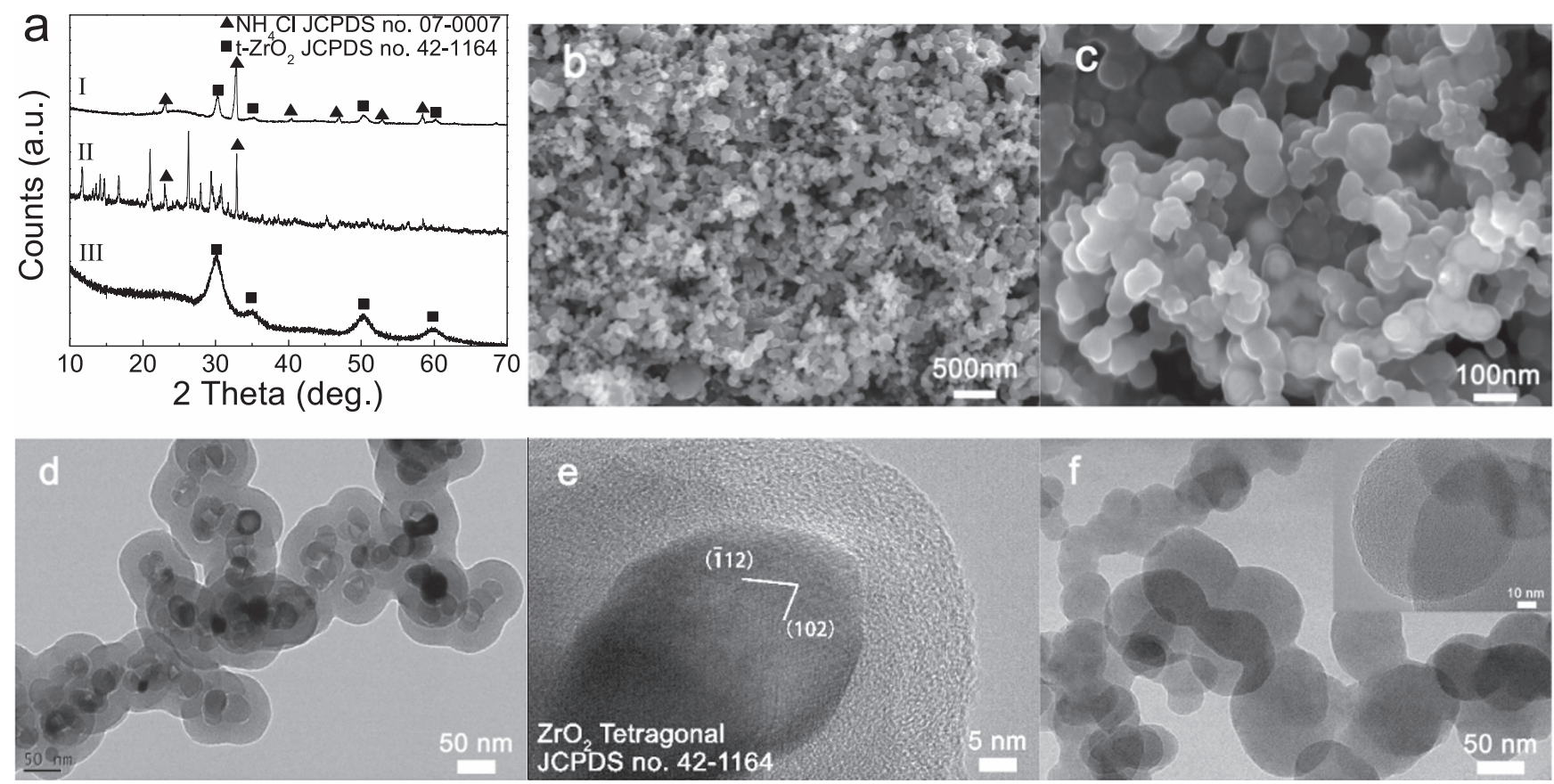

Figure 5. (a) XRD patterns of the powder in sample \#5 in different positions in the autoclave: (I) top; (II) bottom; (III) washed bottom. (b), (c) SEM images of the top powder at different magnifications. (d)-(f) TEM and HRTEM images of the top powder. Inset is the HRTEM image of the amorphous solid carbon.

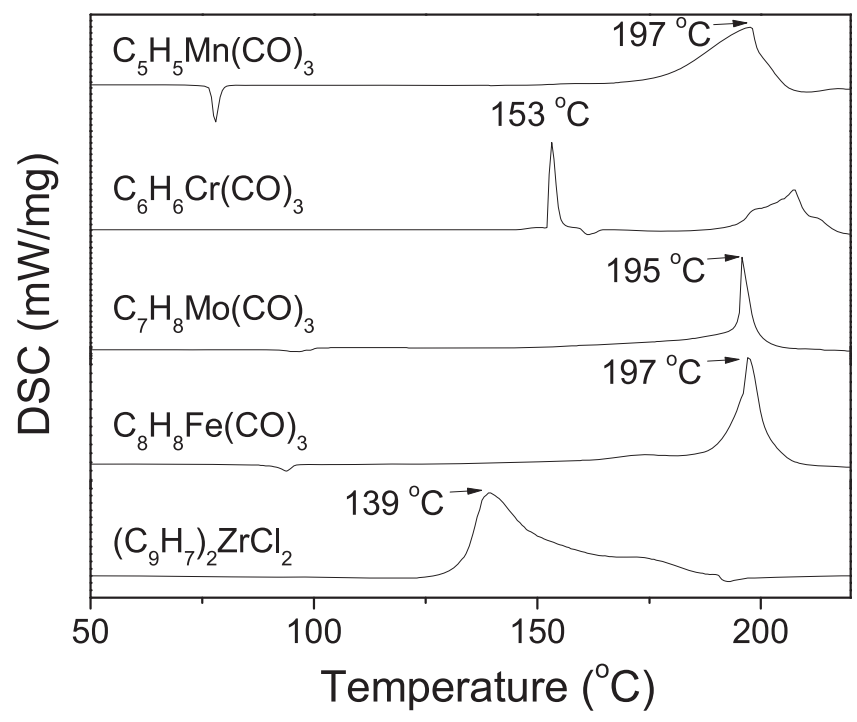

Figure 6. DSC curves of different samples in sealed pans.

strong exothermic peak for each sample. The severe oxidation reaction should be caused by the oxidation of cyclic ligands by $\left(\mathrm{NH}_{4}\right)_{2} \mathrm{~S}_{2} \mathrm{O}_{8}$ and releases large amounts of heat as the hydrogen atoms are transformed into $\mathrm{H}_{2} \mathrm{O}$. As a result, a schematic of the oxidation synthesis process is proposed in figure 7 . The mixture of the metal $\pi$-complex and $\left(\mathrm{NH}_{4}\right)_{2} \mathrm{~S}_{2} \mathrm{O}_{8}$ are unreactive at low temperature and are firstly placed in the bottom of the autoclave (figure 7(a)). When the temperature increases to the reaction temperature, the oxidation reaction takes place rapidly and produces blast water vapour, which can push some reactants to the top of the autoclave (figure 7(b)). Additionally, a large amount of heat is liberated during the reaction, significantly raising the inner temperature

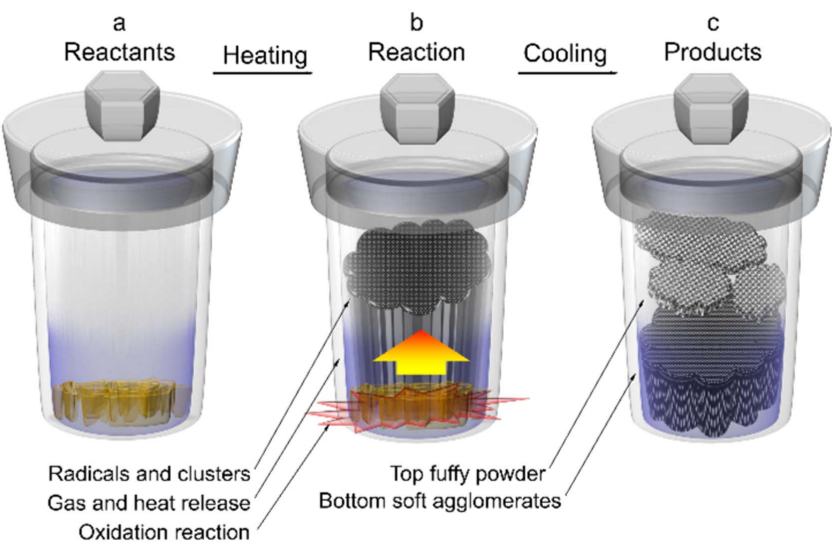

Figure 7. The schematic of the oxidation synthesis process.

of the sealed reactor, which is much higher than the reaction temperature [15]. The high temperature will inevitably result in the cleavage of metal $\pi$-complex, and then the generation of different kinds of clusters and radicals, and eventually the formation of a carbon shell and the metal oxide/sulfide nanocrystals. For samples \#1 and \#4, both metal oxide and sulfide nanocrystals are found in the products, demonstrating that $\mathrm{O}$ and $\mathrm{S}$ radicals are simultaneously generated during the drastic exothermic reaction. Therefore, the weight percentage content of metal oxide and sulfide nanocrystals should depend on the ratio of $\mathrm{O}$ to $\mathrm{S}$ radicals in the autoclave. Otherwise, some oxophilic metal atoms will prefer to bond with oxygen atoms to form stable metal oxides (samples \#2, \#3 and \#5). The carbon clusters from the cleaved cyclic ligands surround these nanocrystals to form a core-shell structure. However, carbon-carbon bonds of benzene are quite stable and consequently unreactive under many reaction conditions [20]. The 
strong oxidizing agent and high temperature must be required for the cleavage of benzene rings. The oxidation temperature of sample \#2 is as low as about $153{ }^{\circ} \mathrm{C}$, which is insufficient for the complete $\mathrm{C}-\mathrm{C}$ bond-tearing of the stable benzene ligand into small carbon clusters, leading to the formation of a very thin carbon layer on the $\mathrm{Cr}_{2} \mathrm{O}_{3}$ nanocrystals. For sample $\# 5$, the reaction temperature is only about $139^{\circ} \mathrm{C}$, suggesting that the $\mathrm{C}_{9} \mathrm{H}_{7}$ ligand is unstable and susceptible to oxidation at low temperature. And the $\mathrm{C}_{9} \mathrm{H}_{7}$ molecule can be easily cleaved and carbonized, resulting in the formation of a thick carbon shell. After cooling, a fluffy powder consisting of homogeneous nanoparticles with a core-shell structure is collected, which is formed by the gaseous clusters and radicals in the top of the autoclave (figure 7(c)). The quantity of the generated gas in the reaction is not high and mainly limited by the number of $\mathrm{H}$ atoms in the ligands. Relative to the weight of the metal $\pi$-complex, the generated gas is 22.05-32.11 wt.\% for these samples on the assumption that only $\mathrm{H}_{2} \mathrm{O}$ vapor is formed in the process. This means most of the solid products are deposited in the bottom of the autoclave, corresponding to the soft agglomerates. It has been proved by the XRD patterns that carbon encapsulated nanocrystals and $\left(\mathrm{NH}_{4}\right)_{3} \mathrm{H}\left(\mathrm{SO}_{4}\right)_{2}$ are the major products in the bottom powder. The $\left(\mathrm{NH}_{4}\right)_{3} \mathrm{H}\left(\mathrm{SO}_{4}\right)_{2}$ may also serve as a reactant for the production of ammonium metal sulfides at the reaction temperature, such as $\left(\mathrm{NH}_{4}\right)_{2} \mathrm{Mn}_{2}\left(\mathrm{SO}_{4}\right)_{3}$ and $\left(\mathrm{NH}_{4}\right)_{3} \mathrm{Fe}\left(\mathrm{SO}_{4}\right)_{3}$. On the other hand, the byproduct of $\mathrm{NH}_{4} \mathrm{Cl}$ in sample \#5 is reactive to the metal $\pi$-complex in the oxidation atmosphere, leading to the formation of amorphous carbon particles instead of a core-shell structure, sequentially decreasing the uniformity of the bottom powder $[15,16]$. For samples \#2 and \#3, it is suggested that the oxidation reactions are drastic and that most of the metal $\pi$-complexes are pushed up to the top of the autoclave, and then only the top fluffy powder is obtained.

In summary, it has been proved that carbon encapsulated nanocrystals with a core-shell structure can be commonly synthesized by the strongly exothermic reaction of the metal $\pi$-complex with a strong oxidizing agent, which is independent of the molecular structure of the ligand. More metal $\pi$ complexes with varying numbers of ligands and carbon atoms can be chosen for controlling the carbon content in the coreshell structure and the shell thickness for different applications. It is also a meaningful starting point for the practical application of the method because we can further select cheap organics containing metal atoms and more general organic ligands as precursors instead of the high-price metallocene complexes or metal $\pi$-complexes.

\section{Conclusions}

Several carbon encapsulated nanocrystals with core-shell structure, including $\mathrm{MnS}$ and $\mathrm{MnO} @ \mathrm{C}, \mathrm{Cr}_{2} \mathrm{O}_{3} @ \mathrm{C}$, $\mathrm{MoO}_{2} @ \mathrm{C}, \mathrm{Fe}_{7} \mathrm{~S}_{8}$ and $\mathrm{Fe}_{3} \mathrm{O}_{4} @ \mathrm{C}, \mathrm{ZrO}_{2} @ \mathrm{C}$, are synthesized by a strong exothermic reaction of metal $\pi$-complexes with $\left(\mathrm{NH}_{4}\right)_{2} \mathrm{~S}_{2} \mathrm{O}_{8}$ in an autoclave at below $200{ }^{\circ} \mathrm{C}$. The molecular structure of the metal $\pi$-complex can be destroyed by the generated large amounts of heat during the oxidation of hydrogen atoms in the ligands. The ligands will be cleaved into small carbon fragments, which further transform into an amorphous carbon shell. The central metal atoms are oxidized to metal oxide/sulfide nanocrystals with an equiaxed morphology and small median size. The formation of the core-shell structure is independent of the numbers of ligands and carbon atoms as well as the metal types, implying that any metal $\pi$-complex can serve as a precursor. The synthesis method is common for the preparation of various carbon encapsulated metal oxide/sulfide nanocrystals and shows clear advantages with its simple process parameters, low energy consumption, and high efficiency and yield, which may be attractive for practical applications.

\section{Acknowledgments}

This work is sponsored by Natural Science Foundation of Shanghai (14ZR1419400, 15ZR1420500), National Natural Science Foundation of China (11572326), Opening fund of State Key Laboratory of Nonlinear Mechanics, and Technology Innovation Action of Shanghai (12nm0503100).

\section{References}

[1] Ghosh Chaudhuri R and Paria S 2012 Chem. Rev. 112 2373-433

[2] Gu M et al 2012 ACS Nano 6 8439-47

[3] Shafeeyan M S, Daud W M A W, Houshmand A and Shamiri A 2010 J. Anal. Appl. Pyrol. 89 143-51

[4] $\mathrm{He}$ C, Wu S, Zhao N, Shi C, Liu E and Li J 2013 ACS Nano 7 4459-69

[5] Bystrzejewski M, Łabędź O, Kaszuwara W, Huczko A and Lange H 2013 Powder Technol. 246 7-15

[6] Zhang H, Liang C, Liu J, Tian Z and Shao G 2013 Carbon 55 $108-15$

[7] Zhao B, Jiang Y, Zhang H, Tao H, Zhong M and Jiao Z 2009 J. Power Sources 189 462-6

[8] Wang W, Sa Q, Chen J, Wang Y, Jung H and Yin Y 2013 ACS Appl. Mater. Inter. 5 6478-83

[9] Athanassiou E K, Grass R N and Stark W J 2006 Nanotechnology 171668

[10] Zheng J, Liu Z Q, Zhao X S, Liu M, Liu X and Chu W 2012 Nanotechnology 23165601

[11] Sun X and Li Y 2004 Angew. Chem Int. Ed. 43 597-601

[12] Wu T, Liu Y, Zeng X, Cui T, Zhao Y, Li Y and Tong G 2016 ACS Appl. Mater. Inter. 8 7370-80

[13] Liu H, Kong L-B, Zhang P, Du J, Li X-M, Luo Y-C and Kang L 2014 Ionics 20 15-21

[14] Liu B, Zhong N, Fan C, Zhou Y, Fan Y, Yu S, Zhang F, Dong L and Yin Y 2014 Carbon 68 573-82

[15] Liu B, Fan C, Chen J, Wang J, Lu Z, Ren J, Yu S, Dong L and Li W 2016 Nanotechnology 27075603

[16] Liu B, Jia D, Zhou Y, Feng H and Meng Q 2007 Carbon 45 $1710-3$

[17] Liu B, Zhang F, Wu Q, Wang J, Li W, Dong L and Yin Y 2015 Mater. Chem. Phys. 151 60-5

[18] Liu B, Shao Y, Zhang Y, Zhang F, Zhong N and Li W 2016 J. Nanopart. Res. 18375

[19] Liu B, Fan C, Chen J, Zhou Y, Dong L and Wang J 2015 Mater. Lett. 142 90-3 
[20] Hu S, Shima T and Hou Z 2014 Nature 512 413-5 